\title{
X-ray free-electron laser heating of water at picosecond time scale
}

\author{
Eva Zarkadoula $\odot,{ }^{1}$ Yuya Shinohara $\odot,{ }^{1}$ and Takeshi Egami $\oplus^{1,2,3}$ \\ ${ }^{1}$ Materials Science and Technology Division, Oak Ridge National Laboratory, Oak Ridge, Tennessee 37831, USA \\ ${ }^{2}$ Department of Materials Science and Engineering, The University of Tennessee, Knoxville, Knoxville, Tennessee 37996, USA \\ ${ }^{3}$ Department of Physics and Astronomy, The University of Tennessee, Knoxville, Knoxville, Tennessee 37996, USA
}

(Received 20 October 2021; accepted 9 December 2021; published 10 January 2022)

\begin{abstract}
Split-pulse x-ray photon correlation spectroscopy using x-ray free-electron laser is a promising tool to probe atomic dynamics in liquid and soft-matter in picosecond time scale, which has been accessible only by spectroscopy. However, sample heating by $\mathrm{x}$-ray beam is a major obstacle for this technique. Using molecular dynamics and the two-temperature model we examine the atomic level response of water to x-ray laser pulse at picosecond time scale and compare with observations from recent $\mathrm{x}$-ray free-electron laser experiments. We investigate the effects of the heating due to x-ray laser pulses of different energies and the effects of the heat dissipation on the structure and dynamics of water through the atomic density correlation and the dynamic structure factor. Our results indicate, in agreement with experiment that in addition to the beam energy the time delay between the two pulses is a critical factor for obtaining reliable information on the atomic level dynamics of water.
\end{abstract}

DOI: 10.1103/PhysRevResearch.4.013022

\section{INTRODUCTION}

Understanding the atomic-scale structure and dynamics of liquid is of fundamental importance for controlling the transport properties of liquid. Despite many experimental and computational efforts, a number of fundamental questions remain unanswered. For example, water-one of the fundamental liquids-exhibits various anomalous features [1,2]. To understand their physical origin, the structure and dynamics of water have been studied using x-ray and neutron scattering [3-10], optical spectroscopy [11-13], and computer simulation [14-16]. Elastic x-ray and neutron scattering provide the pair-distribution function (PDF) of liquid by means of Fourier transform of the structure function (SF). The PDF describes the atomic density correlation through the distribution of the distances between atoms, yielding the snap-shot structure of liquid [17]. Optical spectroscopies provide the information on the frequency of inter- and intramolecular motion, but not on its spatial correlation. Recent work on extracting information on the atomic dynamics through inelastic x-ray scattering (IXS) elucidated the dynamic correlations in water through the Van Hove function, $G(r, t)$ [18]. The Van Hove function can be determined as the double Fourier-transform of the dynamics structure factor, $S(Q, E)$, where $Q$ and $E$ are the momentum and energy transfer, which can be measured by IXS. However, with the IXS only the dynamics up to a few ps can be probed because of the limited energy resolution.

$\mathrm{X}$-ray photon correlation spectroscopy (XPCS) using x-ray free electron laser (XFEL) [19] is a potential alternative ap-

Published by the American Physical Society under the terms of the Creative Commons Attribution 4.0 International license. Further distribution of this work must maintain attribution to the author(s) and the published article's title, journal citation, and DOI. proach in studying the dynamics in extended time scales, from femtoseconds to nanoseconds $[20,21]$. By combining XFEL with X-ray split-and-delay optics (SDO) [22-24] or using a finite pulse duration of XFELs [25] and accelerator-based double $\mathrm{x}$-ray pulse generations [26,27], one can extract information, through the speckle contrast, about the intermediate scattering function, $F(Q, t)$, which is the Fourier-transform $(E \rightarrow t)$ of $S(Q, E)$. This achieves experimental access to the dynamics at time and length scales necessary for studying atomic level behavior beyond a few ps, a time scale that is accessible by IXS. In general, it is highly nontrivial to obtain information experimentally on both spatial and temporal atomic correlations through measurements of either the dynamics structure factor or intermediate scattering function. In the XPCS utilizing the SDO, an X-ray pulse generated by a self-seeded $\mathrm{x}$-ray free-electron laser [28,29] is split into set of two subpulses, with one of the subpulses delayed by an extra-path length. The two pulses impinge upon the sample, and scattered speckles are measured by a detector. The correlation between two speckle patterns yields the information on $F(Q, t)$, where $t$ is the time delay of the second pulse. The Fourier transform of $F(Q, t)$, known as the Van Hove function [18], provides the direct information on the interparticle correlations in time and in real space [30].

As demonstrated in Ref. [24] for ps time scale, this method enables measurements of the intermediate function $F(Q, t)$, but the reliability of the measurements can be compromised by the heating effect on the sample by the first pulse. In conventional $\mathrm{x}$-ray studies, the interaction of $\mathrm{x}$-rays with materials such as scattering, absorption, and emission of photoelectrons is assumed to be negligible and thus it is assumed that $\mathrm{x}$-ray irradiation does not change the dynamics and structure of material. This assumption is not valid for a bright femtosecond $x$-ray pulses from XFEL [19]. The interaction of intense $\mathrm{x}$-ray pulses and material at femtosecond time scales 
have been explored both theoretically and experimentally, and nonthermal [31] and thermal (through heat diffusion) [32] melting processes have been observed. The fluence of $x$-ray pulses used in the split-pulse XPCS study is in the order of micro joule per pulse, which is orders of magnitude smaller than those used in the studies of nonthermal melting. Nevertheless, it still provides energy high enough to change the temperature of the sample. The induced heating by the $\mathrm{x}$-ray laser pulses, due to the excitation of electrons in the sample and the subsequent energy transfer due to electron-phonon interactions, cannot be directly measured experimentally. However, in Ref. [24] it is deduced indirectly by the shift in the position of the peak in the measured intensity, $I(Q)$, for each measurement, utilizing the correlation with the temperature dependence of the $Q$ peak position in the SF, $S(Q)$, from earlier x-ray diffraction experiments on water [33]. In general, the main effect of temperature on the SF is to reduce the peak height with increasing temperature, often following the CurieWeiss law [34]. However, in water the peak position of the SF changes with temperature because of the underlying phase change from the low-density water to the high-density water [1,33], which is easier to detect than the height change. Direct measurement of the system temperature is, however, possible in simulation. In particular, the electronic excitations due to an external heat source, such as laser, and the energy deposition and dissipation to the atomic subsystem can be described by the two-temperature model (2T-MD) in molecular dynamics (MD) simulations [35-37]. In this paper, we use the 2T-MD to simulate the $\mathrm{x}$-ray laser pulse interaction with the sample, using water as an example. MD simulations are a very useful tool for accessing the short time scales and length scales of interest for investigating the dynamics of liquids and directly calculating the PDF and the structure function from the spatial correlation of atoms. We investigate the heating effect of the $\mathrm{x}$-ray laser pulse and compare the findings to experiment. Using the trajectories of all atoms during the simulation, we calculate the PDF, and we investigate the short-time atomic dynamics in water through the structure factor.

\section{METHODS}

The MD simulations are performed using the LAMMPS code [38]. The SPC/E (extended simple point charge) water model [39] is used in systems consisting of 31944 atoms (10 648 water molecules), with periodic boundary conditions. The systems are initially relaxed at $310 \mathrm{~K}$ under the constant pressure and constant temperature ensemble (NPT), using a time step of $2 \mathrm{fs}$. The simulations of the $\mathrm{x}$-ray laser heating are performed using the 2T-MD model. In LAMMPS, the 2T-MD is activated using the "fix ttm/mod" implementation [40,41] which accounts for external heat sources, combined with the microcanonical $(N V E)$ ensemble. The time step in the $\mathrm{x}$-ray laser simulations is $1 \mathrm{fs}$. The 2T-MD model is used to simulate the first of the subpulses heating the water in the x-ray splitand-delay-optical setup.

In the 2T-MD model approach, the system is described as two subsystems, the atomic subsystem, and the electronic subsystem, which can exchange heat depending on their local temperature difference [35-37]. The electronic subsystem is modeled as a continuum, and the heat exchange between the electrons and the atomic subsystem takes place via an inhomogeneous Langevin thermostat. The evolution of the electronic temperature $T_{e}$ with an external heat source is described via a heat diffusion equation:

$$
\begin{aligned}
C_{e} \rho_{e} \frac{\partial T_{e}}{\partial t}= & \nabla\left(\kappa_{e} \nabla T_{e}\right)-g_{p}\left(T_{e}-T_{a}\right) \\
& +g_{s} T_{a}^{\prime}+\theta\left(x-x_{\text {surface }}\right) I_{0} \exp \left(-\frac{x}{l_{\text {skin }}}\right) .
\end{aligned}
$$

In this equation, $C_{e}$ is the electronic specific heat, $\rho_{e}$ is the electronic density, $\kappa_{e}$ is the electronic thermal conductivity, $g_{p}$ is the electron-phonon coupling strength, and $g_{s}$ is the electron stopping coupling parameter. $T_{a}$ is the lattice temperature and $T_{a}^{\prime}$ accounts for energy lost from atoms due to the electronic stopping and it has temperature units. $\theta(x)$ is the Heaviside step function, $I_{0}$ is the absorbed laser pulse intensity and $l_{\text {skin }}$ is the depth of skin-layer.

The electronic specific heat depends on the electronic temperature $T_{e}$, with this dependence expressed as

$C_{e}=C_{0}+\left(a_{0}+a_{1} X+a_{2} X^{2}+a_{3} X^{3}+a_{4} X^{4}\right) \exp \left(-(A X)^{2}\right)$,

where $X=T_{e} / 1000$. The dependence of the electronic thermal conductivity $K_{e}$ on the electronic temperature is expressed as $K_{e}=D_{e} \times \rho_{e} \times C_{e}$, where $D_{e}$ is the electronic diffusivity and $\rho_{e}$ is the electronic density. The energies of the x-ray laser beam vary from 2 to $12 \mu \mathrm{J}$, with a step of $2 \mu \mathrm{J}$. The value for the electron-phonon coupling parameter for water is taken from Ref. [42]. Given the low heat conductivity of water $C_{0}$ is taken to be $1 \mathrm{~J} \mathrm{~cm}^{-3} \mathrm{~K}^{-1}$ and the electronic diffusivity is assumed to be $2 \mathrm{~cm}^{2} / \mathrm{s}$ [43]. The $\mathrm{x}$-ray laser pulse duration was $6 \mathrm{fs}$, same as the experimental pulse duration. Since the objective in this study is to investigate atomic dynamics in picoseconds, the time scale explored in the x-ray experiments [24], the simulation time for each run was 2 ps. The wavelength used was taken from the experiment and it is equal to $1.24 \AA$.

The PDF and the SF were calculated using LiquidLib [44]. The PDF describes the distribution of distances between pairs of atoms or the probability of finding a particle $j$ at distance $r$ from a reference particle $i$ :

$$
g(r)=\frac{1}{4 \pi \rho N r^{2}} \sum_{i, j} \delta\left(r-\left|\boldsymbol{r}_{i}-\boldsymbol{r}_{j}\right|\right),
$$

where $\rho$ is the density, $N$ the number of atoms in the system and $r_{i}$ is the position of the ith atom. The PDF can be used to describe the local structure of a liquid at given times.

The SF is related to $g(r)$ through the Fourier transform:

$$
S(Q)=\frac{1}{N} \sum_{i, j} \exp \left(-i \boldsymbol{Q}\left|\boldsymbol{r}_{i}-\boldsymbol{r}_{j}\right|\right) .
$$

For the calculation of $g(r)$ and $S(Q)$, the trajectories of all atoms of the systems were saved at intervals of 1 step ( $1 \mathrm{fs}$ ) for short simulations times $(<0.5 \mathrm{ps})$ and 20 steps $(20 \mathrm{fs})$ for simulation times larger than $0.5 \mathrm{ps}$. A total of 30 simulations, five simulations for each of the x-ray laser pulse energies, were performed. 
The split-pulse XPCS measurement using self-seeded $\mathrm{x}$ rays was carried out BL3, SACLA (Japan). The detailed setting and the analyses procedure are described in Ref. [24]. By changing the path length difference between the two branches in the SDO, the delay time $\Delta t$ between the subpulses were controlled between 0 and 2 ps. Each pulse energy of the subpulses was monitored by the intensity monitors [45] in the SDO system.

\section{RESULTS AND DISCUSSION}

In x-ray scattering experiments, the contributions from the correlation between the oxygen atoms are more dominant due to the larger number of electrons in oxygen compared to hydrogen [10] and therefore the measurements and analyses are based on the oxygen-oxygen (OO) pairs. Simulations gives the benefit of being able to look into the PDFs and the SFs of all pairs. The PDF for OO, hydrogen-hydrogen (HH) and hydrogen-oxygen (HO) pairs, for a representative set of simulations of 2 and $12 \mu \mathrm{J}$ x-ray laser beam energy, which are the lowest and the highest energy used in this work are shown in Fig. S1 in the Supplemental Material [46]. The PDFs at 0, 0.2, and 2 ps are plotted for both energies, as well as the difference between the PDF at 0 and $0.2 \mathrm{ps}$ and 0 ps and 2 ps for clarity. For $2 \mu \mathrm{J}$, the difference between PDFs at different times is very small. No difference is detectable for the $\mathrm{HH}$ pairs, which are very light atoms, while some spikes of small height can be seen at the first peaks of $\mathrm{OO}$ and $\mathrm{HO}$ pairs, showing the effect of heavier oxygen atoms, the PDF of which is experimentally detectable in x-ray experiments, in contrast to the PDFs of $\mathrm{HH}$ and $\mathrm{HO}$ pairs. For $12 \mu \mathrm{J}$, where more energy is available to dissipate through the system, the differences between the PDFs at different times, and therefore the density correlations, are more profound. In Fig. S2 of the Supplemental Material, the PDFs for the 2 and $12 \mu \mathrm{J}$ energy are plotted together for each pair at 0.2 and at 2 ps. The difference of the PDFs is also shown for clarity. As seen here, at $0.2 \mathrm{ps}$ the energy of the beam has no effect on the PDF. At 2 ps, where the energy has dissipated further into the system increasing its temperature, the energy affects the PDF at short distances $r$.

In Fig. S3, the SFs for OO, $\mathrm{HH}$ and $\mathrm{HO}$ pairs are shown, for the same set of simulations of $2 \mu \mathrm{J}$ and $12 \mu \mathrm{J}$ x-ray laser beam energy as shown in Fig. S1 and S2. The SFs of the system at 0.2 and 2 ps are plotted for both energies, and for clarity, the difference between the two is also provided for each pair. While for $2 \mu \mathrm{J}$ energy the PDFs at different times do not change, the higher energy of $12 \mu \mathrm{J}$ results in differences at low $Q$. Especially for the SF of the $\mathrm{OO}$ pairs, the higher energy, and therefore the higher temperature of the system, affects the peak sharpness at low $Q$.

For comparison between the two energies, the SFs for these two $\mathrm{x}$-ray laser beam energies, the lowest $(2 \mu \mathrm{J})$ and the highest $(12 \mu \mathrm{J})$, are plotted together in Fig. S4, at 0.2 and $2 \mathrm{ps}$ simulation time. Their difference is also plotted for all pairs and energies. For 0.2 ps energy the SFs at different times do not change, and at the higher energy of 2 ps results in differences at low $Q$. Especially for the SF of the OO pairs, it can be seen that the higher energy, and therefore the higher temperature of the system, affect the peak sharpness at low $Q$.
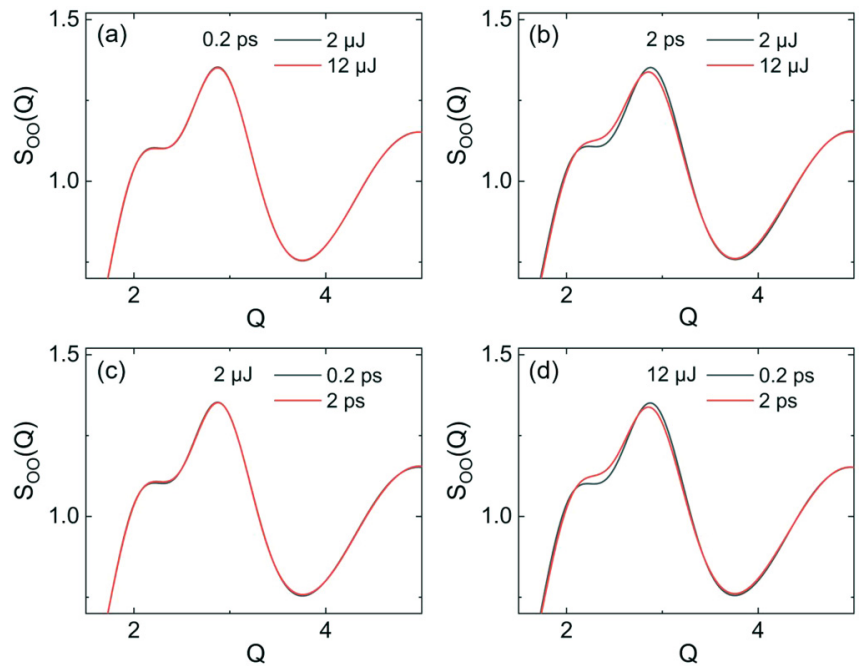

FIG. 1. The SFs for low $\mathrm{Q}$ values for oxygen-oxygen pairs for 2 $\mu \mathrm{J}$ and $12 \mu \mathrm{J}$ at (a) $0.2 \mathrm{ps}$ and (b) 2 ps. (c) The SFs for low $\mathrm{Q}$ values for oxygen-oxygen pairs for $2 \mu \mathrm{J}$ at 0.2 and $2 \mathrm{ps}$. (d) The SFs for low $\mathrm{Q}$ values for oxygen-oxygen pairs for $12 \mu \mathrm{J}$ at 0.2 and $2 \mathrm{ps}$.

In Fig. 1, we focus on the first peaks (low $Q$ values) of the oxygen-oxygen $S(Q)$, since the OO interactions are dominant in the x-ray scattering measurements [10]. Figures 1(a) and 1(b) show the SF at 0.2 and $2 \mathrm{ps}$, respectively, for 2 and $12 \mu \mathrm{J}$. Here the effect of the difference in the $\mathrm{x}$-ray laser beam energy is shown, affecting the peaks sharpness and height. Figures 1(c) and 1(d) show the SF for 2 and $12 \mu \mathrm{J}$, respectively, at 0.2 and 2 ps. Here, the effect of the time delay between the two pulses is shown, where longer delay affects the SF, as the sharpness of the peak and the height are affected.

The temperature evolution for 2 and $12 \mu \mathrm{J}$ energy, for the same systems discussed above, is shown in Fig. 2 for 2 ps simulation time. For $2 \mu \mathrm{J}$, there is a small rise in the temperature of the system, while for $12 \mu \mathrm{J}$, the temperature rises faster and to larger values. For $12 \mu \mathrm{J}$, more energy is transferred to liquid compared to the deposited energy from 2 $\mu \mathrm{J}$, which dissipates further through the phonons (through the atomic subsystem). As seen in Figs. S1-S4, the temperature

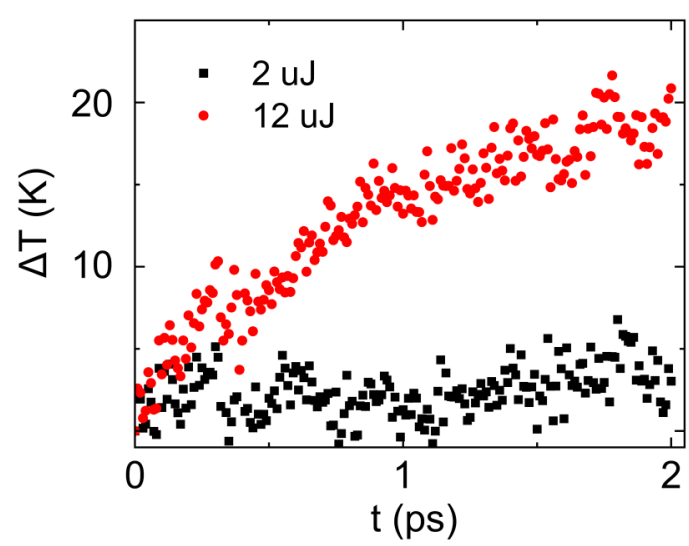

FIG. 2. Temporal evolution of the temperature of water during interaction with $\mathrm{x}$-ray laser beam of $2 \mu \mathrm{J}$ (black squares) and $12 \mu \mathrm{J}$ (red circles) energy during 2 ps simulation time. 


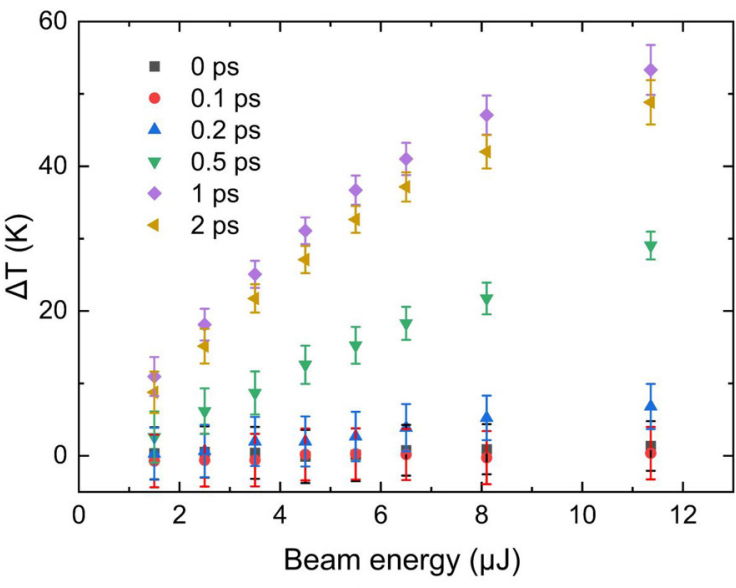

(a)

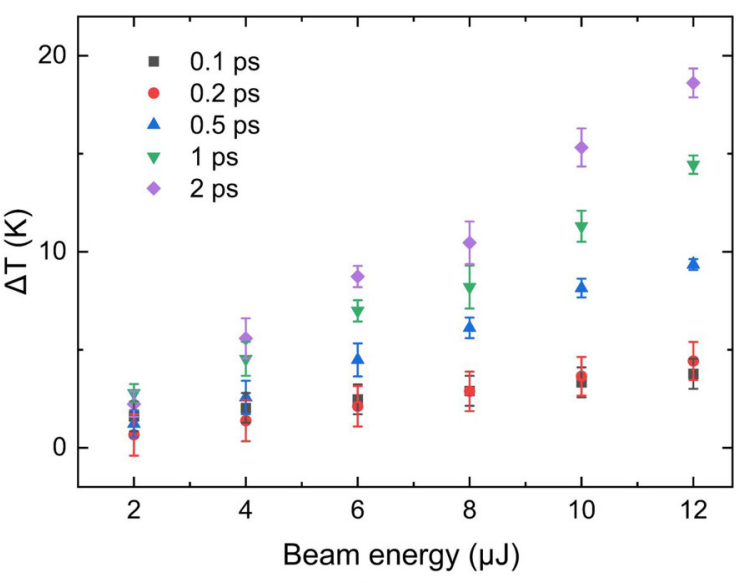

(b)

FIG. 3. (a) Experimentally estimated values of the temperature rise due to different delay times $\Delta(\mathrm{t})$ between the first and the second pulse. The data are reproduced from Ref. [24]. The error bars were determined by the standard deviation of the fitting of the peak position (see Ref. [24] for details on the error calculation). (b) Induced temperature rise in the system obtained from MD simulations due to different energies at different simulation times $\delta$ t. The bars represent the standard error for five simulations.

increase of about $20 \mathrm{~K}$, due to the interaction of the first $\mathrm{x}$-ray laser pulse with water, does not significantly affect the overall PDF and the SF, but the first peaks of the OO SF can be affected, depending on the energy and the time delay $\Delta(t)$ between the first and the second pulse is applied. The first peak of SF mainly reflects the higher-order peaks of PDF, thus medium range order and the coherence of density correlations, indicating that the heating due to the x-ray laser influenced the coherence in the coarse-grained density fluctuations.

During the experiments the time delay $\Delta(t)$ between the first pulse, which excites electrons, and the second pulse varied between 0 and 2 ps. Specifically, the second pulse was applied at $0,0.1,0.2,0.5,1$, and 2 ps after the first pulse. As shown above, the energy of the first pulse may affect the PDF, $g(r)$, and the SF, $S(Q)$. Additionally, the time delay between the two pulses can also affect the reliability of the measurements [24]. Figure 3(a) shows the temperature rise in the system due to the first $\mathrm{x}$-ray laser pulse, for different energies and for different $\Delta(t)$, extracted experimentally. Here the temperature rise was estimated by the shift in the peak position around $Q=2 \AA^{-1}$ by using the temperature dependence of peak position [10]. As seen here, a heating effect is observed for $\Delta(t)$ larger than $0.5 \mathrm{ps}$ and energy larger than 3 $\mu \mathrm{J}$, indicating that for $\Delta(t)$ less than $0.5 \mathrm{ps}$ and energy smaller than $3 \mu \mathrm{J}$ the measurements are not affected by temperature effects.

Figure 3(b) shows the temperature difference $\Delta T$ calculated from the MD simulations of the interaction of water with an $\mathrm{x}$-ray laser pulse, for different beam energies, at different simulation times $\delta t$. For time smaller than $0.5 \mathrm{ps}$, the $\Delta T$ rise is small for small energies, less than $4 \mu \mathrm{J}$, and it reaches a plateau as the energy increases. As the time increases, for 1 and $2 \mathrm{ps}$, the system temperature rises faster and to larger values. These observations indicate that, similarly to the experimental observations, short time delays of the second pulse (up to $0.5 \mathrm{ps}$ ) and low energies (up to $4 \mu \mathrm{J}$ ) do not result in a temperature rise that could significantly affect the density fluctuation measurements. The heat dissipation through the atomic system results in heating effects, and potentially unreliable measurements of the structure factor, for dissipation length larger than about $1 \mathrm{ps}$ (simulation time larger than $1 \mathrm{ps)}$. It can be seen that the temperature differences from the simulations are lower than the experimentally calculated. This is due to approximations made both in experiment and simulation. In experiment, the temperature difference is obtained indirectly; the $Q$ peak position dependence on the beam energy is obtained in Ref. [24] and it is combined with data of the $Q$ peak position dependence on temperature from Ref. [33], in order to acquire the temperature difference as a function of the beam energy, as shown in Fig. 3(a). In the simulations, the 2T-MD model is an approximation which describes very well the heat exchange processes that take place between the atomic and electronic subsystems and while it allows for inclusion of the role of the electronic subsystem, it can also result to quantitative discrepancies such as the one seen here.

In the aforementioned setup, the information obtained nearly immediately after the application of the second pulse concerns the effect of the water and $\mathrm{x}$-ray laser interaction due to the first pulse. To understand the heating effect in splitpulse setups where another probe, e.g., femtosecond optical spectroscopy, is used after the second pulse, we simulated the interaction of two pulses with water assuming that time elapses between the second pulse and the measurement. The energy used for each pulse is $12 \mu \mathrm{J}$ and the time delay between the two pulses is 2 ps. The total simulation time is 4 ps. Figure 4(a) shows the temperature evolution of water due to the interaction with two pulses. The energy deposited from the second pulse at 2 ps results in increased heating. The effect of this heating can be seen in Figs. 4(b) and 4(c) which show the comparison of the PDF and the SF for the OO pairs after the first and after the second pulse. The heating due to the second pulse results in changes within distance of about $6 \AA$ in $g(r)$. The difference is more profound in the height of the first peak, 


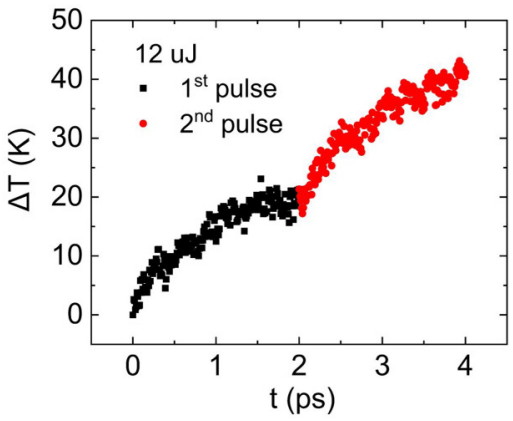

(a)

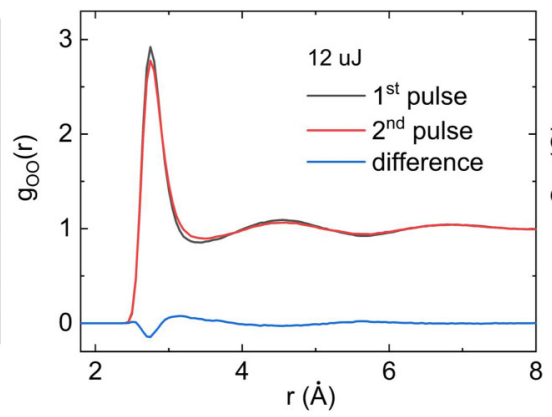

(b)

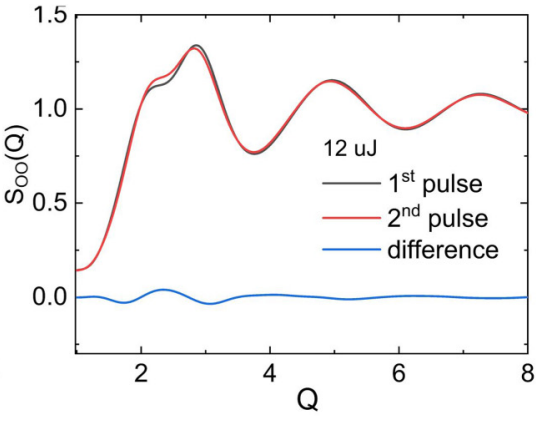

(c)

FIG. 4. (a) Temperature evolution of the system after two pulses of $12 \mu \mathrm{J}$ energy with 2 ps time delay ( 4 ps total simulation time). b) The PDFs for low $Q$ values for oxygen-oxygen pairs after the first pulse, after the second pulse and their difference are shown. (c) The SFs for low $\mathrm{Q}$ values for oxygen-oxygen pairs after the second pulse and their difference are shown.

showing that the second pulse results in increased disorder. A smaller difference between the PDF for the first and second pulse is also observed at the second peak, which is indicative of number or pairs at distances of second nearest neighbors. This density change is reflected in the sharpness on the peaks at low $Q$, in SF as seen in Fig. 4(c). These differences observed here indicate that the time delay between pulses, and therefore the available time for heat dissipation before additional energy is deposited in the system due to the second pulse, is important for obtaining reliable results and measurements.

\section{CONCLUSIONS}

Using molecular dynamics simulations, we investigate the heating of water due to x-ray free-electron laser beam interaction and the impact of the temperature rise in the atomic level response in terms of the pair distribution function and the structure function. The beam energy ranges from 2 to $12 \mu \mathrm{J}$, at short times up to $2 \mathrm{ps}$. For the split-pulse technique the effect of the heat dissipation between two pulses is studied and compared to recent observations from recent $\mathrm{x}$-ray free-electron laser experiments. Our findings indicate that while split-and-delay optics can enable measurements of the dynamic structure factor at high $Q$ values, the time delay and the beam energy can significantly compromise the quality of the measurements, which is in agreement with experiment.
Molecular dynamics simulations are a useful tool for the fundamental understanding of the short time- and length-scales processes, in the order of a few angstroms and picoseconds, respectively, that take place in split-pulse experiments and can provide insights for the advancement of techniques accessing the atomic level dynamics in liquids.

The Department of Energy will provide public access to these results of federally sponsored research in accordance with the DOE Public Access Plan [47].

\section{ACKNOWLEDGMENTS}

The work was supported by the U. S. Department of Energy (DOE), Office of Science, Office of Basic Energy Sciences, Materials and Sciences and Engineering Division. The experiments at SACLA were carried out under the approval of JASRI (Proposals No. 2018B8041, No. 2019A8043, and No. 2019B8011). This research used resources of the Compute and Data Environment for Science (CADES) at the Oak Ridge National Laboratory, which is supported by the Office of Science of the U.S. DOE under Contract No. DEAC05-00OR22725 and the resources of the National Energy Research Scientific Computing Center (NERSC), a U.S. DOE Office of Science User Facility located at Lawrence Berkeley National Laboratory, operated under Contract No. DE-AC0205CH11231.
[1] A. Nilsson and L. G. M. Pettersson, The structural origin of anomalous properties of liquid water, Nat. Commun. 6, 8998 (2015).

[2] P. Ball, Water: Water-an enduring mystery, Nature (London) 452, 291 (2008).

[3] A. H. Narten and H. A. Levy, Liquid water-molecular correlation functions from x-ray diffraction, J. Chem. Phys. 55, 2263 (1971).

[4] A. Sette, J. Alexander, and H. M. Grey, Interaction of antigenic peptides with $\mathrm{Mh}_{\mathrm{c}}$ and $\mathrm{T}_{\mathrm{cr}}$ molecules, Clin. Immunol. Immunop. 76, S168 (1995).

[5] G. Monaco, A. Cunsolo, G. Ruocco, and F. Sette, Viscoelastic behavior of water in the terahertz-frequency range: An inelastic x-ray scattering study, Phys. Rev. E 60, 5505 (1999).
[6] G. Ruocco and F. Sette, The high-frequency dynamics of liquid water, J. Phys.: Condens. Mat. 11, R259 (1999).

[7] A. K. Soper and M. A. Ricci, Structures of High-Density and Low-Density Water, Phys. Rev. Lett. 84, 2881 (2000).

[8] G. Hura, J. M. Sorenson, R. M. Glaeser, and T. Head-Gordon, A high-quality $\mathrm{x}$-ray scattering experiment on liquid water at ambient conditions, J. Chem. Phys. 113, 9140 (2000).

[9] C. Huang et al., The inhomogeneous structure of water at ambient conditions, Natl. Acad. Sci. USA 106, 15214 (2009).

[10] L. B. Skinner, C. C. Huang, D. Schlesinger, L. G. M. Pettersson, A. Nilsson, and C. J. Benmore, Benchmark oxygen-oxygen pair-distribution function of ambient water from X-ray diffraction measurements with a wide Q-range, J. Chem. Phys. 138, 074506 (2013). 
[11] C. J. Fecko, J. D. Eaves, J. J. Loparo, A. Tokmakoff, and P. L. Geissler, Ultrafast hydrogen-bond dynamics in the infrared spectroscopy of water, Science 301, 1698 (2003).

[12] T. Tokushima, Y. Harada, O. Takahashi, Y. Senba, H. Ohashi, L. G. M. Pettersson, A. Nilsson, and S. Shin, High resolution $\mathrm{x}$-ray emission spectroscopy of liquid water: The observation of two structural motifs, Chem. Phys. Lett. 460, 387 (2008).

[13] J. S. Hansen, A. Kisliuk, A. P. Sokolov, and C. Gainaru, Identification of Structural Relaxation in the Dielectric Response of Water, Phys. Rev. Lett. 116, 237601 (2016).

[14] F. H. Stillinger and T. A. Weber, Inherent structure in water, J. Phys. Chem-Us 87, 2833 (1983).

[15] E. Shiratani and M. Sasai, Molecular scale precursor of the liquid-liquid phase transition of water, J. Chem. Phys. 108, 3264 (1998).

[16] D. C. Elton and M. Fernandez-Serra, The hydrogen-bond network of water supports propagating optical phononlike modes, Nat. Commun. 7, 10193 (2016)

[17] B. E. Warren, X-Ray Diffraction (Addison-Wesley, Reading, 1969).

[18] L. Van Hove, Correlations in space and time and born approximation scattering in systems of interacting particles, Phys. Rev. 95, 249 (1954).

[19] B. W. J. McNeil and N. R. Thompson, X-ray free-electron lasers, Nat. Photonics 4, 814 (2010).

[20] G. Grubel, G. B. Stephenson, C. Gutt, H. Sinn, and T. Tschentscher, XPCS at the European x-ray free electron laser facility, Nucl. Instrum. Meth. B 262, 357 (2007).

[21] C. Gutt, L. M. Stadler, A. Duri, T. Autenrieth, O. Leupold, Y. Chushkin, and G. Grubel, Measuring temporal speckle correlations at ultrafast x-ray sources, Opt. Express 17, 55 (2009).

[22] T. Osaka, T. Hirano, Y. Sano, Y. Inubushi, S. Matsuyama, K. Tono, T. Ishikawa, K. Yamauchi, and M. Yabashi, Wavelengthtunable split-and-delay optical system for hard $\mathrm{x}$-ray freeelectron lasers, Opt. Express 24, 9187 (2016).

[23] W. Roseker et al., Towards ultrafast dynamics with split-pulse $\mathrm{x}$-ray photon correlation spectroscopy at free electron laser sources, Nat. Commun. 9, 1704 (2018).

[24] Y. Shinohara, T. Osaka, I. Inoue, T. Iwashita, W. Dmowski, C. W. Ryu, Y. Sarathchandran, and T. Egami, Split-pulse x-ray photon correlation spectroscopy with seeded x-rays from x-ray laser to study atomic-level dynamics, Nat. Commun. 11, 6213 (2020).

[25] F. Perakis et al., Coherent x-rays reveal the influence of cage effects on ultrafast water dynamics, Nat. Commun. 9, 1917 (2018).

[26] T. Hara et al., Two-colour hard x-ray free-electron laser with wide tunability, Nat. Commun. 4, 2919 (2013).

[27] A. Marinelli et al., High-intensity double-pulse x-ray freeelectron laser, Nat. Commun. 6, 6369 (2015).

[28] I. Inoue et al., Generation of narrow-band x-ray free-electron laser via reflection self-seeding, Nat. Photonics 13, 319 (2019).

[29] Y. Sun, M. Dunne, P. Fuoss, A. Robert, D. Zhu, T. Osaka, M. Yabashi, and M. Sutton, Realizing split-pulse x-ray photon correlation spectroscopy to measure ultrafast dynamics in complex matter, Phys. Rev. Research 2, 023099 (2020).
[30] T. Egami and Y. Shinohara, Correlated atomic dynamics in liquid seen in real space and time, J. Chem. Phys. 153, 180902 (2020).

[31] I. Inoue et al., Atomic-Scale Visualization of Ultrafast Bond Breaking in X-Ray-Excited Diamond, Phys. Rev. Lett. 126, 117403 (2021).

[32] R. J. Husband et al., x-ray free electron laser heating of water and gold at high static pressure, Commun. Mater. 2, 61 (2021).

[33] L. B. Skinner, C. J. Benmore, J. C. Neuefeind, and J. B. Parise, The structure of water around the compressibility minimum, J. Chem. Phys. 141, 214507 (2014).

[34] C. W. Ryu, W. Dmowski, K. F. Kelton, G. W. Lee, E. S. Park, J. R. Morris, and T. Egami, Curie-Weiss behavior of liquid structure and ideal glass state, Sci. Rep. 9, 18579 (2019).

[35] D. M. Duffy and A. M. Rutherford, Including the effects of electronic stopping and electron-ion interactions in radiation damage simulations, J. Phys.: Condens. Mater. 19, 016207 (2006).

[36] E. Zarkadoula, D. M. Duffy, K. Nordlund, M. A. Seaton, I. T. Todorov, W. J. Weber, and K. Trachenko, Electronic effects in high-energy radiation damage in tungsten, J. Phys. Condens. Mater. 27, 135401 (2015).

[37] E. Zarkadoula, G. Samolyuk, and W. J. Weber, Twotemperature model in molecular dynamics simulations of cascades in Ni-based alloys, J. Alloy Compd. 700, 106 (2017).

[38] S. Plimpton, Fast Parallel Algorithms for Short-Range Molecular-Dynamics, J. Comput. Phys. 117, 1 (1995).

[39] P. Mark and L. Nilsson, Structure and dynamics of the TIP3P, SPC, and SPC/E water models at 298 K, J. Phys. Chem. B 105, 9954 (2001).

[40] J. K. Chen, D. Y. Tzou, and J. E. Beraun, A semiclassical twotemperature model for ultrafast laser heating, Int. J. Heat Mass. Trans. 49, 307 (2006).

[41] G. E. Norman, S. V. Starikov, V. V. Stegailov, I. M. Saitov, and P. A. Zhilyaev, Atomistic modeling of warm dense matter in the two-temperature state, Contrib. Plasm. Phys. 53, 129 (2013).

[42] M. A. Flores-Mancera, J. S. Villarrubia, and G. Massillon, Electron inelastic mean free paths for $\mathrm{LiF}, \mathrm{CaF}_{2}, \mathrm{Al}_{2} \mathrm{O}_{3}$, and liquid Water from $433 \mathrm{keV}$ down to the energy gap, ACS Omega 5 , 4139 (2020).

[43] M. Toulemonde, C. Dufour, A. Meftah, and E. Paumier, Transient thermal processes in heavy ion irradiation of crystalline inorganic insulators, Nucl. Instrum. Meth. Sec. B 166, 903 (2000).

[44] N. P. Walter, A. Jaiswal, Z. K. Cai, and Y. Zhang, LiquidLib: A comprehensive toolbox for analyzing classical and ab initio molecular dynamics simulations of liquids and liquid-like matter with applications to neutron scattering experiments, Comput. Phys. Commun. 228, 209 (2018).

[45] K. Tono, T. Kudo, M. Yabashi, T. Tachibana, Y. P. Feng, D. Fritz, J. Hastings, and T. Ishikawa, Single-shot beam-position monitor for x-ray free electron laser, Rev. Sci. Instrum. 82, 023108 (2011).

[46] See Supplemental Material at http://link.aps.org/supplemental/ 10.1103/PhysRevResearch.4.013022 for the PDF and SF for all pairs, for a representative set of simulations of $2 \mu \mathrm{J}$ and $12 \mu \mathrm{J}$ $\mathrm{x}$-ray laser beam energy.

[47] http://energy.gov/downloads/doe-public-access-plan. 